\title{
Organic Gas Sorption on Plasma-Polymerized Allylamine Films Coated with Quartz Crystal Microbalance
}

\author{
Hidenobu Aizawa ${ }^{1, *}$, Tetsuro Muramatsu ${ }^{2}$, Kazutoshi Noda ${ }^{1}$, Ryuichi Naganawa ${ }^{1}$, \\ Shigeru Kurosawa ${ }^{1}$, Ryutaro Tsuruyama ${ }^{2}$ and Shinji Sunohara ${ }^{2}$ \\ ${ }^{1}$ Reserach Institute for Environmental Management Technology, National Institute of Advanced Industrial \\ Science and Technology (AIST), 16-1 Onogawa, Tsukuba, Ibaraki 305-8569, Japan \\ ${ }^{2}$ Dalton Corporation, 13 Ichigaya-Hachiman-cho, Shinjuku-ku, Tokyo 162-0844, Japan
}

Keywords: plasma-polymerized allylamine films, quarts crystal microbalance, pollant release and transfer register (PRTR), volatile organic compounds (VOCs), gas absorption properties, $\mathbf{N}$, $\mathbf{N}$-dimethyl formaamide

\section{Introduction}

Recently, the amount of organic gas emission released into the atmosphere has increased. According to the pollutant release and transfer register (PRTR) data of FY2009, 60\% of the total amount of emissions are volatile organic compounds (VOCs) [1]. Since the generation of harmful photochemical oxidants and suspended particulate matter is detrimental to living systems, the emission of VOCs into the atmosphere is a significant environmental problem, and hence, reduction in the amount of organic gas emission is required [2]. Control of effluent concentration varies with the type of VOC. VOC concentration is analyzed using instrumental analysis methods such as gas chromatography-mass spectrometry (GC-MS). However, GC-MS is expensive and real-time measurement using this method is difficult [3]. Therefore, a VOC analysis method that can perform highly-precise real-time measurements is required. Removal and control can be facilitated if VOC concentration is understood correctly. Quartz crystal microbalance (QCM) is a high-accuracy technique for detecting gas concentration in real time [4]. In QCM sensors, polymer films [5-8] and inorganic materials are employed as established materials for detecting VOCs [9]. Plasma polymerization has also been employed conventionally to fabricate thin functional films that can facilitate surface modification of materials [10]. Plasma-polymerized ( $\mathrm{pp}$ ) films adhere firmly to most substrates and are highly resistant to chemical and physical treatments. In this study, we develop VOC measuring techniques that use a QCM and pp films [11-13] and aim to construct a sensor that can provide real-time and highly precise measurement.
In the preparation of a pp-film, amino groups such as ethylenediamine, diaminopropane, butylamine, heptylamine, aniline, diaminocyclohexane, and acetonitrile are used. In particular, allylamine is the most commonly utilized organic precursor [14]. The performance of devices constructed with amine-containing plasma polymers depends strongly on their composition. Amino-group-containing plasma polymers can be utilized in numerous applications, e.g., modification of wastewater purification membranes [15], carbon nanotubes [16], and polymer microspheres [17]; improvement in biocompatibility of artificial materials and immobilization of biomolecules for biomedical applications [18]; QCM sensor fabrication $[19,20]$; surface plasmon resonance sensor [21]; and surface transverse wave devices [22]. This paper focuses on the evaluation of gas sorption properties of pp-Allylamine (AA) films using 18 commonly analyzed gases.

\section{Experimental}

We employed guaranteed grade reagents. For plasma polymerization, model BP-1 from Samco Co. (Kyoto, Japan) was used. QCM with polished gold electrodes (AT-cut of $9 \mathrm{MHz} ; 8 \times 8 \times 0.185$ $\mathrm{mm}^{3}$ ) was purchased from Nihon Dempa Kogyo Co. Ltd. (Tokyo, Japan). Fig. 1(a) shows the schematic of the pp equipment and Fig. 1 (b) shows the position settings of four QCMs on the lower electrode. The pp-AA films were deposited on both sides of QCM by polymerization for the duration of $1 \mathrm{~min}$ each side. The annealing treatment for $12 \mathrm{~h}$ at $10 \mathrm{~Pa}$ and $80^{\circ} \mathrm{C}$ removed the stress of the pp-AA films. The plasma polymerization process was followed as described previously. 
Oscillating frequencies of 16 QCMs were measured using a laboratory-made 16-channel sensor array measurement system (Fig. 2). The RF power of glow discharge is an important controllable parameter in plasma polymerization.
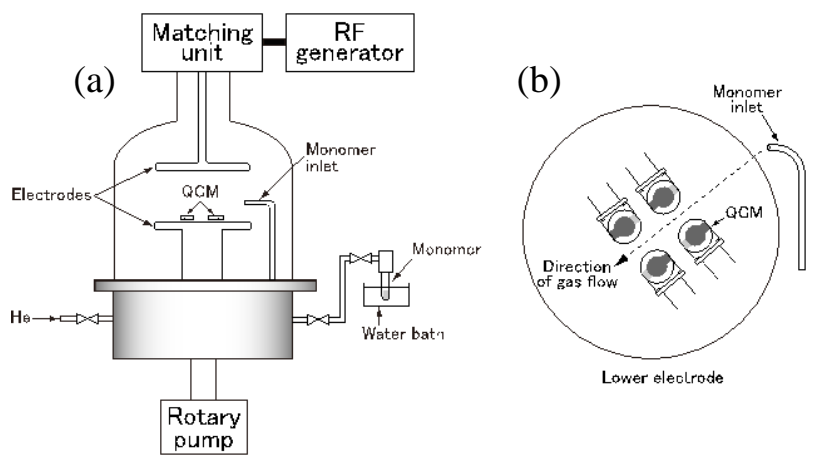

Fig. 1 (a) Schematic of pp equipment. (b) Position settings of four QCMs for pp-AA film coated on the lower electrode.

The RF powers were settled at 90,110, 130 and $150 \mathrm{~W}$ and vapor pressure of the monomer was settled at $100 \mathrm{~Pa}$.

We specifically examined the relationship between the chemical properties of pp-AA films plasma polymerized under various RF powers and the gas sorption properties of pp-AA-film-coated QCMs with 18 different gases (ethanol, methanol, isopropyl alcohol, tetrahydrofuran, ethyl ether, ethyl acetate, formaldehyde, acetone, hexane, benzene, dichloromethane, chloroform, toluene, N,Ndimethylformamide, 1,4-dioxane, triethylamine, acetic acid, and acetonitrile).

The analyte gases were generated by a gas detector tube (GASTEC Co., Ltd., Kanagawa, Japan).

\section{Result and discussion}

The film thickness of pp-AA was calculated from the frequency shift of QCM using Equation 1:

$$
\Delta \mathrm{F}=-2.26 \times 10^{-7} \cdot \mathrm{F}_{0}^{2} \cdot \rho \cdot \mathrm{T},
$$

where $\Delta \mathrm{F}$ is the frequency shift of QCM caused by the attached mass of the pp-AA film, $\mathrm{F}_{0}$ is the fundamental oscillating frequency, $\rho$ is the density of a monomer, and $\mathrm{T}$ is the thickness of the pp-AA film.

The gas sorption of analyte gases by the pp-AA film was performed by a 16-channel QCM sensor array (Fig. 2).

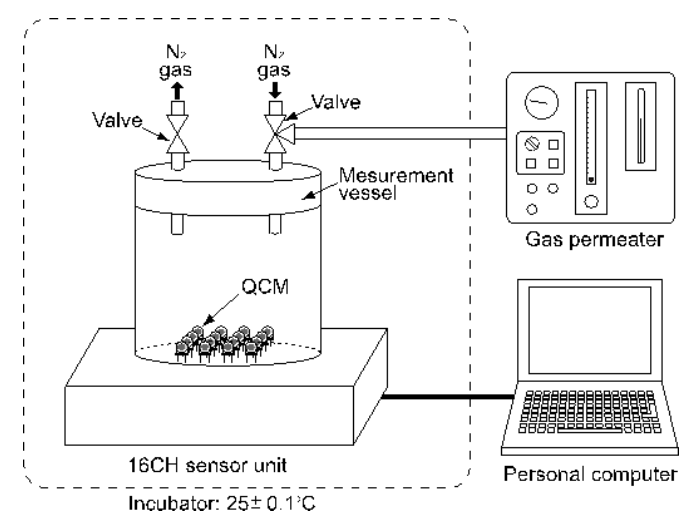

Fig. 2 Schematic diagram of laboratory-made 16-channel QCM sensor array for gas sorption measurement.

First, $\mathrm{N}_{2}$ gas $(1 \mathrm{~L} / \mathrm{min})$ was used to purge the measurement vessel. Next, nitrogen gas flow was stopped and the inflow was changed to the analyte gas. Analyte gases were prepared using Permeater (PD-1, GASTEC Co., Ltd.), a gas generator for calibration purposes that utilizes permeation and diffusion tubes to generate the calibration gas. The analyte gas flowed through the reaction container at flow velocity $0.2 \mathrm{~L} / \mathrm{min}$ and measured the frequency change caused by gas adsorption for 20 min. Subsequently, desorption of the analyte gas was performed by the inflow of nitrogen gas to the reaction chamber for $20 \mathrm{~min}$.

Table 1 Concentration of analyte gas generated with the Permeater.

\begin{tabular}{cc}
\hline Analyte gas & Concentration $(\mathrm{ppm})$ \\
\hline Ethanol & 330 \\
Methanol & 950 \\
Isopropyl alcohol & 207 \\
Tetrahydrofuran & 804 \\
Ethyl ether & 558 \\
Ethyl acetate & 385 \\
Formaldehyde & 176 \\
Acetone & 1269 \\
Hexane & 511 \\
Benzene & 225 \\
Dichloromethane & 1344 \\
Chloroform & 865 \\
Toluene & 108 \\
N,N-Dimethylformamide & 18 \\
1,4-Dioxane & 163 \\
Triethylamine & 207 \\
Acetic acid & 87 \\
Acetonitrile & 560 \\
\hline
\end{tabular}

Concentrations of the analyte gas were generated with the Permeater (Table 1). The response sensitivity was calculated by Equation 2: 


$$
R S=\frac{G / M_{1}}{P / M_{0}} \times \frac{1}{C},
$$

where $R S$ is the response sensitivity to the analyte gas $\left(\mathrm{ppm}^{-1}\right), \mathrm{P}$ is the amount of pp-AA film coated on QCM (ng), $\mathrm{M}_{0}$ is the molecular weight of an allylamine monomer $(\mathrm{g} / \mathrm{mol}), \mathrm{G}$ is the sorption amount of the analyte gas (ng), $\mathrm{M}_{1}$ is the molecular weight of the analyte gas, and $\mathrm{C}$ is the concentration of the analyte gas (ppm). The response sensitivity of each analyte gas is shown in Fig. 3. The response sensitivities of the pp-AA film to each analyte gas are in the order ethanol, methanol, isopropyl alcohol, tetrahydrofuran, ethyl ether, ethyl acetate, formaldehyde, acetone, hexane, benzene, dichloromethane, chloroform, toluene, $\mathrm{N}, \mathrm{N}$-dimethylformamide, 1,4-dioxane, triethylamine, acetic acid, and acetonitrile.

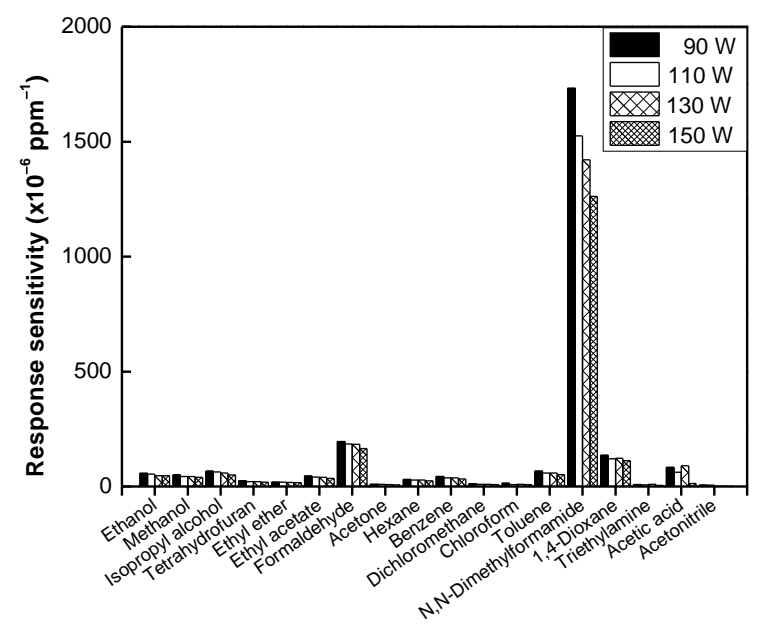

Fig. 3 Response sensitivity of pp-AA films coated with QCMs to apply organic gases.

\section{Conclusion}

The pp-AA-film-coated QCM measured the adsorption of each organic gas. Response sensitivity of the pp-AA films for the 18 different analyte gases was different and in the order $\mathrm{N}, \mathrm{N}$-dimethylformamide $>$ formaldehyde > 1,4-dioxane > acetic acid > isopropyl alcohol > toluene > ethanol > methanol > ethyl acetate > chloroform > dichloromethane $>$ acetone > triethylamine $>$ acetonitrile. The high response sensitivity to the $\mathrm{N}, \mathrm{N}$-dimethylformamide and formaldehyde in which a pp-AA film has an aldehyde group was shown.

\section{Acknowledgments}

This study was financially supported by Dalton Corporation. Part of this work was conducted at the Nano-Processing Facility, supported by IBEC Innovation Platform, AIST.

\section{References}

1. Ministry of the Environment of Japan; http://www.env.go.jp/chemi/prtr/result/gaiyo_H21 /2_summary_2_1_4.pdf

2. S. Himeno, K. Okubo, S. Fujita, Kagaku Kogaku Ronbunshu, 32 (2006) 79.

3. T. Seko, K. Usukura, N. Onda, Jpn. Analyst, 52 (2003) 1215.

4. J. M. Slater, J. Paynter, Analyst, 119 (1994) 191.

5. M. Matsuguchi, T. Uno, T. Aoki, M. Yoshida, Sens. Actuators, B, 131 (2008) 652.

6. S. Ichinohe, H. Tanaka, Y. Kanno, Sens. Actuators, B, 123 (2007) 306.

7. G. N. Tchoupo, A. G. Elie, Sens. Actuators, B, 110 (2005) 81.

8. S. V. Patel, T. E. Mlsna, B. Fruhberger, E. Klaassen, S. Cemalovic, D. R. Baselt, Sens. Actuators, B, 96 (2003) 541.

9. H. Sugimoto, H. Tanaka, Y. Kanno, Jpn. J. Appl. Phys., 47 (2008) 637.

10. H. Yasuda, Plasma Polymerization; Academic Press: New York (1985) 432.

11. S. Kurosawa, H. Miura, H. Takahashi, J. W. Park, H. Aizawa, K. Noda, K. Yamada, M. Hirata, Sens. Actuators, B, 108 (2005) 558.

12. H. Aizawa, S. Kawashima, S. Kurosawa, K. Noda, T. Fujii, M. Hirata, Thin Solid Films, 515 (2007) 4141.

13. S. Kurosawa, T. Hirokawa, K. Kashima, H. Aizawa, J. W. Park, M. Tozuka, Y. Yoshimi, K. Hirano, J. Photopolym. Sci. Technol., 15 (2002) 323.

14. A. Choukourov, H. Biederman, D. Slavinska, L. Hanley, A. Grinevich, H. Boldyryeva, A. Mackova, J. Phys. Chem. B, 109 (2005) 23086.

15. R. C. Ruaan, T. H. Wu, S. H. Chen, J. Y. Lai, J. Membr. Sci., 138 (1998) 213.

16. Q. Chen, L. Dai, M. Gao, S. Huang, A. Mau, $J$. Phys. Chem. B, 105 (2001) 618.

17. A. Denizli, B. Salih, E. Piskin, J. Chromatogr., A, 773 (1997) 169.

18. J. Kim, H. Park, D. Jung,S. Kim, Anal. Biochem., 313 (2003) 41.

19. K. Nakanishi, H. Muguruma, I. Karube, Anal. Chem., 68 (1996) 1695.

20. S. Kurosawa, N. Kamo, H. Aizawa, M. Muratsugu, Biosens. Bioelectron., 22, (2007) 2598.

21. S. Sasaki, E. Kai, H. Miyachi, H. Muguruma, K. Ikebukuro, H. Ohkawa, I. Karube, Anal. Chim. Acta, 363 (1998) 229.

22. H. Yatsuda, M. Nara, T. Kogai, H. Aizawa, S. Kurosawa, Thin Solid Films, 515 (2007) 4105. 